\title{
Barriers to Conducting Multicenter Nursing Research
}

Lisa S. Lewis ${ }^{1^{\star}}$, Molly McNett ${ }^{2}$, Julia Aucoin ${ }^{3}$, Kristina Riemen ${ }^{4}$, Susan Yeager ${ }^{5}$ and DaiWai M Olson ${ }^{5}$

${ }^{1}$ Lisa S. Lewis, Nursing Instructor, Watts School of Nursing, 2828 Croasdaile Drive, Suite 200, Durham, North Carolina, USA

${ }^{2}$ Molly McNett, PhD, RN, CNRN, Director, Nursing Research, MetroHealth Medical Center, Nursing Business Office, 2500 MetroHealth Drive, Cleveland, Ohio, USA

${ }^{3} J u l i a$ Aucoin, DNS, RN-BC, CNE, Nurse Research Scientist, Duke University Health System, Durham, North Carolina, USA

${ }^{4}$ Kristina Riemen, CCRC, Clinical Research Coordinator, Division of Neurology, Box 2900, Duke University Medical Center, 225 Bryan Research Building, Duke University, Durham, North Carolina, USA

${ }^{5}$ Susan Yeager MS, RN, CCRN, ACNP, Neurocritical Care Nurse Practitioner-Lead, Clinical Instructor Acute Care Nurse Practitioner, Wexner Medical Center at The Ohio State University, The Ohio State University College of Nursing, 7th floor, Room 790, 395 W. 12th Avenue, Columbus, Ohio, USA

${ }^{6}$ DaiWai M. Olson PhD RN CCRN, Associate Professor of Neurology and Neurotherapeutics, Associate Professor of Neurosurgery, University of Texas Southwestern, 5323 Harry Hines, BIvd, Dallas, Texas, USA

${ }^{*}$ Corresponding author: Lisa S. Lewis, Nursing Instructor, Watts School of Nursing, 2828 Croasdaile Drive, Suite 200, Durham, North Carolina, USA, Tel: 919-672-2608; Fax: 919-383-4014; E-mail : lisa.lewis@duke.edu

Received date: April 2, 2014; Accepted date: April 18, 2014; Published date: April 22, 2014

Copyright: (C) 2014 Lewis LS, et al. This is an open-access article distributed under the terms of the Creative Commons Attribution License, which permits unrestricted use, distribution, and reproduction in any medium, provided the original author and source are credited.

\begin{abstract}
Introduction: The move towards evidence-based nursing practice requires active participation by nurses at all levels along the continuum of care. Despite this fact, nursing involvement in multicenter research is limited. This not only limits the generalizability of findings, but hinders collaborative and interdisciplinary research networking between hospitals. Reasons for single site nursing research barriers have been explored. Yet, knowledge of the barriers nurses face when they seek to engage in multicenter research activities is limited.
\end{abstract}

Methods: A core group of experienced nurse researchers sought to initiate a multicenter observational research study of intracranial pressure monitoring. This article uses a case series approach to highlight the challenges encountered throughout the process.

Results: Barriers to multicenter nursing research were identified and categorized by theme.

Conclusions: Institutional changes are suggested to support the participation of clinical nurses in research and recommendations are made for future study.

Keywords: Nursing research; Multicenter research; Barriers

\section{Introduction}

A key element in advancing the ability of nurse clinicians to become actively engaged in research is a fuller understanding of the barriers that nurses face in completing multicenter studies. This paper will also present a rationale for the value of clinical nurses to participate within the research infrastructure of their organization, particularly in multicenter research. The progression of nursing research roles is such that, while studies should be led by a skilled nurse researcher, our professional responsibility suggests that clinical staff can be developed beyond the data collection role. The Study of Intracranial Pressure (ICP) Monitoring in Critically Ill (SIM City) is described, and a detailed discussion of the barriers that clinical nurses identified in the course of the conduct of the study provided. As there was no prospective planning or comparisons for control, this collection, through anecdote and survey, of experiences of sites participating in a nursing-led multicenter observational study is described as a case series. The purpose of this paper is to highlight specific barriers identified in the conduct of a nursing-led multicenter trial and to present potential solutions for overcoming these challenges $[1,2]$.

\section{Background and Significance}

Nurses have been conducting and publishing highly relevant research findings as far back in history as the Crimean War [3]. Presently, the American Nurses Credentialing Center (ANCC) Magnet Recognition Program ${ }^{\circledR}$ standards include requirements for nursing research [4]. Healthcare institutions have moved towards embracing the concept of Evidence-Based Practice (EBP). As more nurses become educated in EBP there is an expectation that more registered nurses (RNs) will be involved in research. In addition to Magnet $^{\circ}$ requirements, the National League for Nursing has placed emphasis on multicenter research studies as reflected by support with funding [5]. This is due to the fact that multicenter nursing research provides greater generalizability [5].

The literature includes reports of challenges with multicenter research studies, especially in relation to barriers associated with ethics approval, in other disciplines with clearer professional expectations of participation in research. Doctorally-prepared nurse researchers, as traditionally defined, have a role not only in discovery but in mentorship. A nursing-led multicenter clinical research study is an appropriate opportunity to mentor interested clinical nurses who are novices in research. The expectation that clinical nurses will participate in research is a relatively new one and discussion of the 
barriers associated with nursing-led multi-center clinical research studies is limited.

\section{Literature Review}

\section{Barriers to nursing research}

Many articles present barriers to research utilization, a topic much studied including the development and use of a tool to assess obstacles to nurses using evidence in their practice, the BARRIERS scale $[6,7]$. Fewer articles can be found on the barriers to nurses participating in research. An integrative literature review demonstrated that it was uncommon for nurse researchers to report methodological barriers or cite specific challenges to completing Randomized Clinical Trials (RCTs) when they published research reports [8]. Some barriers to nursing research are consistently described in the literature. Derbyshire [9] described the "unholy trinity" of barriers to nursing research, indicating that a lack of funding, inadequate protected time, and lack of education/exposure are common barriers to nurse-led research. Nurses conduct research in environments that are often not supportive, engaging in activities on their personal time without compensation, and with limited knowledge or experience of the research process. There is an expectation that the role of the nurse, whether staff or advanced practice, is focused on clinical practice and research activities are not emphasized or often appreciated [10]. Nurses frequently report that great personal motivation and personal sacrifices are necessary to integrate clinical research into their professional role. Research activities are often on one's own time, in addition to other professional responsibilities [7,11]. Two related themes were that of nurses' lack of ownership in the research in which they participate and the lack of dissemination of results of nursing research. Traditionally, much nursing research has involved clinical practice nurses serving as research staff for an academic or medical researcher, collecting data for little recognition or appreciation [12].When nurses have done their own research, they have often lacked the support or encouragement to publish the results [7].

\section{Ethics review barriers}

Challenges associated with the process of obtaining ethics approval are a common theme in the research literature, both nursing and nonnursing, and relating to single site and multicenter studies. One study done in the United Kingdom described experiences of nurses doing clinical research, with nurses frustrated and delayed by bureaucratic processes similar to the nursing research committees and institutional review boards (IRBs) in the United States [11]. Nurse researchers interviewed by Snelgrove and James [11] were suspicious that they were not offered the same institutional support for their research as researchers from other professions. Woodward, Webb [7], also in the United Kingdom, found that nurses perceived their ethics committees to not be understanding of nursing research and to be biased toward medical research [7]. These nurses often were required to present and defend their research proposals to a nursing research committee prior to going through the ethics committee review process, and they found this two-tiered procedure to be burdensome and redundant [7]. While institutions or ethics committees may have good reason for making the research approval process so complex, they did not make such justification clear to the nurses who wanted to do research. These barriers may be more profound when multiplied by the numerous institutions in a multicenter study.

\section{Multicenter research barriers}

Lindquist, Treat-Jacobson [13] stressed the importance of multicenter research in nursing and identified the many benefits of multicenter nursing research, which included recruitment of larger and more diverse samples, faster accrual rates, and the potential for additional subgroup analyses of the dataset. The authors described challenges associated with multicenter research in nursing centered primarily on the design and development of the research protocol, such as identifying ways to control for variation in nursing practices among institutions, standardizing the protocol, ensuring access to a representative and adequate sample, and controlling costs. The issue of ethics approval is discussed by Flynn [14], who points out that the processes used by most IRBs are often not compatible with multicenter research. The current IRB process in the United States was developed in the 1970s when the standard for research was the single site study involving local participants [15].

Gold and Dewa [16] explored the problem of ethics approval in multicenter studies from an interdisciplinary perspective, finding that the current process of approval by the IRB of each institution was wasteful and discouraging to both collaboration and multicenter research in general. They performed a literature search to quantify the costs associated with the multiple IRB approval process in time, money and opportunity, concluding with a call for a centralized, single IRB process for multicenter studies. The centralized IRB review idea has been trialed by the National Institutes of Health (NIH) and the United States Food and Drug Administration (FDA) in some oncology clinical trials, but no definitive process currently exists [17].

A common theme related to nursing research in single site or multicenter trials is the need for persistence and support. A novice nurse researcher described her experience in doing clinical research and suggested that tenacity is the key to overcoming barriers [18]. Several authors found that nursing research is best accomplished when mentors are present and a team approach is used to provide support for getting projects off the ground and maintaining momentum $[6,12]$. Vedelo and Lomborg [8] described a successful Nursing Research Fellowship program at their community hospital that gave staff nurses the opportunity to lead clinical research, with guaranteed paid time off the unit and mentorship and support from a doctorally-prepared nurse and statistician. Schmelzer, et al. [19] described experiences doing a multicenter clinical research study, emphasizing the need for team support and realistic expectations regarding challenges like the IRB process.

While barriers to nursing research are clearly identified, and tools or processes for overcoming those barriers suggested, there is still inadequate information in the literature to support the nurse struggling to accomplish studies in the clinical setting. Vedelo and Lomborg [8] advised nurse researchers performing randomized controlled trials to spread the news about their experiences, positive and negative. "They should publish their results even if they are not statistically significant and describe factors that may have inhibited the desired outcome" (p. 199). While knowledge of potential barriers will not eliminate them, it may encourage the nurse researcher to seek the needed support to persist in overcoming obstacles.

\section{The SIM City Study}

SIM City was designed to explore the variety of nursing interventions, independent (i.e. patient positioning) and interdependent (i.e. administering medications prescribed by a 
Page 3 of 7

physician) that were associated with taking care of patients with ICP monitoring. This was a descriptive study which originated from clinical practice. The genesis for SIM City was a discussion about different approaches used to treat ICP. The study design was developed by a group of nurses attending the 2009 Neurocritical Care Society (NCS) annual meeting in Baltimore, Maryland. The nurses in the NCS include staff RNs, advanced practice nurses (clinical nurse specialist, nurse practitioners), nursing faculty and nurse-scientists (PhD-RN).

The cadre of NCS nurses at the 2009 meeting recognized that best practices and standards exist for ICP monitoring during traumatic brain injury (TBI), and adhering to these standards is associated with improved outcomes [20,21]. Despite this knowledge, variation between institutions and care providers still exist [22]. Because the impact of nursing care was not described in the majority of studies that explored ICP treatment, the decision was made to add to the body of evidence by providing a description of current nursing practice from a multicenter perspective. The SIM City study was designed by doctoral and masters-prepared nurses experienced in research with previous experience collaborating on a multicenter research study [23].

SIM City proposed enrollment at fifty sites around the US. Each site was to enroll two patients with ICP monitoring and also enroll the nurse caring for the patient during the study period. Each nursepatient dyad was observed for two hours by the site coordinator, who documented the frequency and effect of nursing interventions on patient ICP.

The primary research team, comprised of a nurse scientist, an expert clinician and a study coordinator, submitted for ethics approval through the coordinating organization's IRB in March 2009. IRB approval was obtained in July 2009. Recruitment of additional sites for the study was begun using snowball technique among members of the NCS. Criteria for site selection were not explicitly defined; however, criteria for patient subject selection included patients diagnosed with intracranial pathology and an ICP monitoring device in situ, and criteria for nurse subject selection included RNs that were permanent members of the staff of an ICU. Study site recruitment was achieved using fliers at national meetings and via NCS nursing listserv and nursing committee teleconference communication. The response was enthusiastic. An array of nurses ranging from associate-degree staff nurses, to nurse managers, to advanced practice nurses voiced their interest in participating in the SIM City trial and serving as site coordinators and principal investigators (PIs) if their organization allowed their role to do so.

Schmelzer, et al. [19] stated that a multicenter study is a good opportunity for inexperienced investigators to participate in research because the protocol and supporting documents have been developed by the primary research team. The primary research team for SIM City concurred with this belief, and encouraged novice nurse researchers to participate.

The primary research team was required by their institution's IRB to send interested parties an email invitation. Only the hospitals/RNs who responded to that email were recorded as potential SIM City sites. Between July of 2009 and December of 2011, clinical nurses from 46 different hospitals affirmed an interest in participating and received copies of study materials (study protocol, patient consent form, and nurse consent form). Twenty-two of these sites received IRB approval prior to January 1, 2012. Of these, data from 28 nurse/patient dyads was received by the completion of the study.

\section{Barriers}

As the SIM City study progressed, it became evident to the primary research team that this seemingly straightforward study was being hindered by a variety of unexpected challenges or barriers. Initially, the challenges were reported by the clinical site coordinators (who were PIs if permitted by their site IRB) and aggregated by the primary research team. As the challenges mounted and seemingly similar themes were recognized, a more formalized inquiry was initiated. This was achieved by email survey from the primary research team to all of the clinical site coordinators, whether they had already reported barriers or not (Table 1).

1) Was SIM the first study you ever had to submit for an IRB review?

2) Did your hospital have Magnet designation at the time of IRB submission of the SIM study?

3) Did you experience extreme (i.e. greater than usual) difficulty getting the SIM study approved by IRB or other committees? If so, please describe.

Table 1: Data Collection Tool

Site coordinators were asked if they had previous experience submitting research proposals for IRB approvals; if they experienced any difficulties obtaining the necessary approvals for this study; and if their organization was Magnet ${ }^{\ominus}$ designated by the ANCC. Site coordinators that reported difficulties obtaining IRB or other institutional approvals were then asked to elaborate on their experiences and seventeen sites responded specifically to this query. While this limited response raises a concern of selection bias in the survey results, data collected provided additional perspective on the barriers to the SIM City study. Six of the seventeen site coordinators (35\%) reported extreme difficulty with obtaining approval for this research study in their institution. Of these six coordinators reporting extreme barriers, five were new investigators without prior experience submitting research for IRB approval. It was not asked if these site coordinators had any training regarding the IRB process. Among these five, two were still able to obtain IRB approval for the SIM study, while the remaining three novice investigators reported extreme difficulty and were unable to obtain final IRB approval for the study at their institutions. There was one additional investigator who had prior experience with submitting research for IRB approval that still reported unexpected difficulties obtaining approval for this multicenter study. While novice researchers of all professions experience challenges in navigating the IRB process, the nurses involved in SIM City were subject to some extraordinary barriers.

To consider the barriers encountered in the SIM City study from another perspective, data was compiled for the number of days each site needed to achieve six milestones: agreeing to participate in SIM City, receiving study documents from the primary site, submitting to IRB, obtaining IRB approval, completing data collection on the first dyad, and completing data collection on the second dyad. Table 2 provides the range, mean, median and 50th interquartile range (IQR) for each of these milestones. 


\begin{tabular}{|l|l|l|l|l|}
\hline \multirow{2}{*}{ Milestones } & \multicolumn{4}{l}{ Number of Days } \\
\cline { 2 - 5 } & Range & Mean & Median & IQR \\
\hline $\begin{array}{l}\text { Agreed to participate } \\
\text { after invite letter sent }\end{array}$ & $0-324$ & 18.78 & 1 & $0-3$ \\
\hline IRB documents sent & $0-324$ & 22.54 & 2 & $1-7$ \\
\hline Submitted to IRB & $0-255$ & 123.81 & 117 & $\begin{array}{l}85-176.7 \\
5\end{array}$ \\
\hline Obtained IRB Approval & $88-486$ & 240.30 & 209 & $132-352$ \\
\hline Obtained first dyad & $151-742$ & 412.21 & 394 & $\begin{array}{l}332.5-47 \\
9.75\end{array}$ \\
\hline
\end{tabular}

Collaboration

Table 3: Barriers Categorized by Framework from Streiner and Sidani (2010)

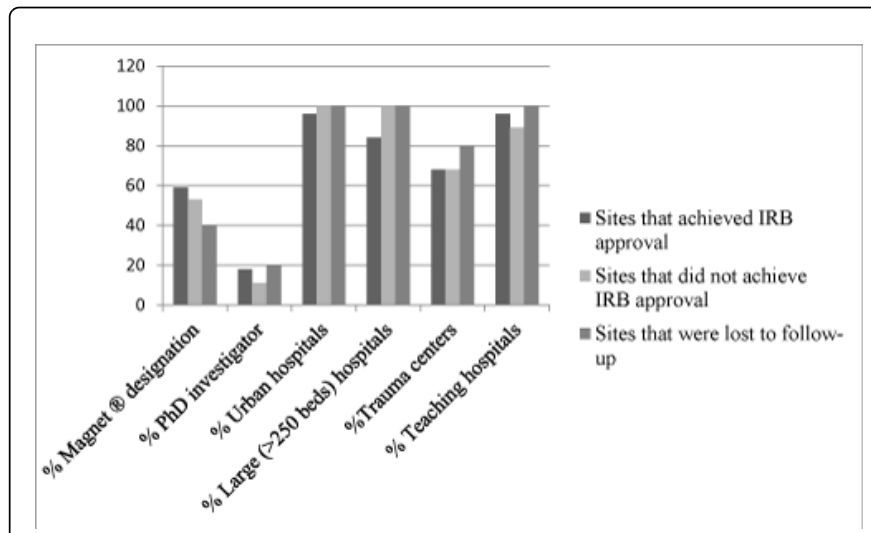

Figure 1: SIM City Site Attributes

data was that of the characteristics of the participating sites. Size and location of the institution, as well as designations such as Magnet status, trauma center, or teaching hospital could have been associated with differences in support for nursing research. The qualifications of the site coordinator-doctorally prepared or not-were also considered. Comparing sites that achieved IRB approval to those that attempted unsuccessfully to gain IRB approval as well as those sites lost to follow up demonstrated surprisingly little variation across groups (see Figure 1). As might be expected in a study of critically ill neurological patients, nearly all sites were large hospitals (defined as greater than 250 beds), in urban areas, that were academic teaching centers. Greater than half of the sites were designated trauma centers. Sites that achieved IRB approval were somewhat more likely to have the Magnet ${ }^{\circledR}$ designation, but few sites in any group had a doctorallyprepared nurse as site coordinator. After consideration from the multiple perspectives described above, the barriers experiences were organized into categories as suggested by Streiner and Sidani [24]: Ethics approval, accessing participants (sites), recruitment and retention (subjects), study implementation, data collection, data analysis, and collaboration. Table 3 lists the identified barriers by those categories. Barriers in the categories of data collection and data analysis were not experienced by the SIM City site coordinators, so are not discussed here (Table 3)

\begin{tabular}{|l|l|}
\hline Category & Barrier \\
\hline Ethics approval & Extensive or atypical IRB requirements \\
\cline { 2 - 2 } & $\begin{array}{l}\text { Inconsistent IRB requirements across } \\
\text { institutions }\end{array}$ \\
\hline Accessing participants or sites & $\begin{array}{l}\text { Hospital unit or department policies or } \\
\text { practices }\end{array}$ \\
\cline { 2 - 2 } & Lost to follow 7up \\
\hline \multirow{2}{*}{$\begin{array}{l}\text { Recruitment and retention of } \\
\text { subjects }\end{array}$} & Low volume of suitable subjects \\
\cline { 2 - 2 } Study implementation & Cultural modifications \\
\hline & Competing priorities \\
\cline { 2 - 2 } & Lack of mentors \\
\cline { 2 - 2 } & Lack of protected space \\
\hline
\end{tabular}


lamented that the IRB process to approve her site's participation in SIM City took seven months, when the average was four weeks for other research studies for which she had gained IRB approval.

Although IRBs follow the same general principles, there are variations in how each IRB conducts business. The purpose of the IRB is human subjects' protection following federal guidelines of the Office of Human Subjects Protection, a division of the US Department of Health and Human Services and the accreditation guidance offered by Association for the Accreditation of Human Research Protection Programs, Inc. In order to comply and be consistent, policies and practices are in place, which require periodic re-examination as roles and technology evolve. Gold and Dewa [16] found dramatic variation among IRBs in multicenter studies including criteria for an expedited or full review, number of changes required per consent form, and number of revisions and resubmissions required within the same multicenter study. The SIM City experience included similar variation in IRB requirements. The time and effort necessary to revise and adapt documents to the site-specific IRB required ongoing enthusiasm and persistence that was not possible for all potential nurse researchers.

The qualifications to be a PI were inconsistent among IRBs. The logic behind not allowing RNs to act in the PI capacity is not documented, but has been noted in previous literature to exist at multiple institutions [25]. Several nurse investigators were required to have a physician involved in the research study as a sponsor or as the PI. One nurse reported that, after successfully recruiting a physician (MD) to serve as PI at her site, she was informed that the MD must be physically present at the IRB meeting; which caused the physician to decline participation and the site withdrew from the study. Alternatively, others were instructed that a $\mathrm{PhD}$ nurse was required to be a member of the study team to demonstrate that the study team was qualified to perform research. Many of these requirements were rooted in system-specific standard operating procedures (SOPs). It soon became evident that there was great variation in navigating these institution-specific SOPs, from PI eligibility to IRB process.

Another example of the variation in SOPs for research surfaced when some site coordinators were instructed by their IRBs that face to face discussions with families of potential study subjects were not permitted until the study was introduced to the family/subject by their MD. This policy was related to system level practices outlined by privacy or risk management departments. While some site coordinators were permitted to approach potential subjects, as long as they were assigned to the care of that patient, others were not. .

Further variations in IRB requirements were observed. How data are recorded for transfer was clear or acceptable to one IRB; it was not acceptable to another. This observational study sometimes provoked questions such as: "Is this within the role of the nurse? How is observation good science? What will the nurse do with the findings since nurses don't control whether the patient undergoes ICP?" Some IRBs weighed in on the science and the human subjects protection, while others focused only on the latter, allowing that the science is managed through an earlier approval. Others had concerns about the sample size of only 100 , not recognizing that the value of 50 sites is significant when looking at variations in clinical practice among institutions. Many IRBs approved for one year and required a continuing renewal report while others saw the risk as minimal and provided approval for the duration of the study. Some IRBs met without the PI being present and others requested attendance at the IRB meeting to respond to specific questions. Finally the IRB meetings varied from weekly to quarterly.
The SIM City study, as unfunded research, may have raised questions about scientific merit as there was not the evidence implicit in funded research that peer review or a rigorous scientific process had occurred in the development of the study. Yet the primary research team had taken the study through nursing science and institutional review prior to other sites applying for IRB approval.

\section{Accessing Participants (sites)}

Finding nurse researchers that were interested in participating in SIM City was not difficult - 46 potential sites were identified during the recruitment period. However, sites that expressed initial interest were sometimes not able to participate in the study due to a variety of barriers at the level of the hospital unit or department.

Some researchers encountered barriers such as the beliefs that every action by a nurse must be compensated, that research is too hard for staff colleagues to support, and that consenting patients in a community hospital will change how patients view the facility. Often the conduct of research is viewed as a professional opportunity and obligation, not requiring additional compensation. Yet some staff nurses are employed in facilities where support from colleagues is difficult because of all activities requiring compensation. Examples of this that occurred during the SIM City study include lack of supervisor permission to come in during non-work hours accompanied by a limitation of not observing another nurse during the potential researcher's own assigned shift. Another nurse shared that the project would require Clinical Practice Council approval. This was delayed because of a constantly full agenda and actual policy changes taking priority. Interpersonal dynamics and organizational politics about who gets to work on projects was a barrier to unit support to even identify a potential dyad. There was also the concern that if the nurses did not perform the ICP monitoring according to standard that it could reflect negatively upon the institution, although the data report would be deidentified.

There were 11 sites lost to follow-up. This attrition rate may be related to a variety of factors. Nurses took new jobs, nurses had their time reprioritized and some nurses simply gave up or lost interest. Many who expressed initial interest did not follow through, without any explanation or further communication.

\section{Recruitment and Retention (subjects)}

Recruitment of subjects for the SIM City study was an area in which minimal barriers were experienced. Subject retention was not an issue in this study, which required only two hours of observation per subject. Some investigators found that identifying the necessary two subjects took longer than they expected, citing low volume of qualified patients at their sites. One investigator felt that enrolling subjects would be difficult without having a Spanish-language consent form, which required additional IRB approval and additional time.

\section{Study Implementation}

Barriers experienced in the category of study implementation included competing priorities, the lack of mentors and the lack of protected space (secure office and personal computer).

\section{Competing Priorities}

Competing priorities were varied. When one hospital came up with a new initiative, the investigator had to deal with that and put 
SIM City on the back burner. Nurses often have a limited window of availability and interest to perform a study. When the work is seen as additive rather than fundamental, then it is easy for a competing agenda to get in the way. One investigator was pulled off the study to assist with The Joint Commission readiness.

Nurses are valued as the constant presence at the bedside. Because of this, time away from patient care duties may be considered nonproductive time and therefore held to fiscal scrutiny. Uncompensated work such is this was considered by many managers to be work that the researchers would do on their own time, and not an expectation of the researcher's job. This resulted in some nurse researchers feeling that they did not have time to carry out the study in addition to all of their other work expectations. Lack of immediate contribution to the organization's balanced scorecard was cited as a reason for not participating in the study by one potential nurse researcher. When nurses are required to absorb the work of research into their clinical time without any additional compensation, they may feel this to be unfair [29].

Given the clinical focus and the multiple distractions experienced by nurses, uninterrupted or any accessibility to email is challenging. This challenge can lead to communication delays and an inability to perform the necessary steps to obtain IRB approval or to comprehensively navigate through unfamiliar hospital processes such as approval from unit-level committees or managers.

\section{Lack of Mentors}

Navigating the process of conducting a research study requires persistence as well as knowledge and skill, all of which have to be developed by any nurse researcher. Guidance from an experienced peer or mentor can facilitate the development of such skills and prevent the novice to research from having to self-learn through trial and error. The lack of available experienced nursing research peers or mentors was one barrier noted. This lack of availability may have been related to the absence of these providers entirely or the overextended workload of those that were present. In one institution, nurses interested in research were asked to present projects to the nurse researcher for approval. However, when the SIM City study was presented, the nurses were asked to limit their research questions to those that involved skin care and falls. These were institutionally supported research topics, and the nurse researcher did not have adequate hours to mentor staff to advance the nursing research program, too.

\section{Lack of Protected Space}

Protected space in which to conduct research proved to be elusive or impossible for some. The majority of clinical nurses lack personal office space and computer access. This makes uninterrupted time to work on research based, intellectual work logistically challenging. This further challenges nurses in ensuring the IRB that they have adequate resources in place to securely store study documents according to regulations. Data confidentiality and integrity is difficult when a secured computer and filing system are not available.

\section{Collaboration}

In this study, collaboration in the process of writing the protocol and planning the study was not problematic. Where collaboration became difficult was during implementation of the study, especially related to communication between the primary research team and the site coordinators.

Throughout the SIM City study, the primary research team attempted to maintain communication and offer support to the site coordinators. Quarterly emails and follow-up phone calls if emails went unreturned were implemented by the primary research team. Often delays in response from the site coordinators occurred, due to competing priorities and other barriers discussed in the study implementation category. The primary research team developed a growing awareness of the barriers the sites were experiencing as the study progressed; the primary team did not always know how to offer support that would be meaningful. This created a barrier in terms of collaboration.

\section{Discussion and Recommendations}

Most of the barriers experienced in the SIM City study have been identified in the literature, some in more detail than others. The challenges posed to SIM City site coordinators in terms of study implementation are perennial difficulties experienced by all nurse researchers. Competing priorities, lack of mentors and lack of protected space are variations on Darbyshire's themes of lack of funding, lack of time and lack of experience [9]. The difficulties in the area of collaboration during SIM City are strongly connected to these barriers.

Ethics approval difficulties are discussed widely in the literature on nursing and health-related research in other disciplines. The concern about variations in IRBs posing obstacles to multicenter studies and calls for new and updated processes for ethics approval are established [16]. Less widespread are reports highlighting how IRBs treat nurseled multicenter research. Some qualitative data from the United Kingdom suggests that nurse-led research is not supported or viewed by IRBs in the same manner as studies initiated by other disciplines $[11,26]$. The SIM City experience fuels the concern that there may be insufficient understanding of nursing research among ethics committees.

Another concern raised by the SIM City experience is that barriers exist to obtaining sites for multicenter nursing research. Potential nurse researchers were unable to participate in SIM City due to limitations in their ability to perform research activities in their work setting, or limitations in taking the study to IRB because of system requirements such the need for a PI with specific qualifications. There are hospitals that do not have nurse scientists, although their population includes critically ill neurologic patients. Supporting clinical nurses who are trying to bring discovery science to the bedside provides an important role for nursing leadership and nurse researchers. While it is a reasonable process to require pre-IRB review of a study presented by an investigator without academic research credentials, it seems that processes are burdensome and discouraging of clinical nursing research in some institutions.

Solutions for some of the identified barriers are suggested by the SIM City experience. In the category of accessing participants, institutional changes would support more sites being able to participate in multicenter studies. Institution-level programs of support for nursing research are inconsistently developed, and could include the nurturing and development of clinical nurses to participate in nursing research Programs such as the ones described by Vedelo and Lomborg [8] and Atkinson, et al. [27] thoroughly address the institutional barriers to nursing research, but even something as basic 
as the development of an established process for clinical nurses to initiate or coordinate research studies would facilitate multicenter studies like SIM City.

Study implementation barriers are widespread, and have a common theme of research not being a priority component of the nurse's role. Promoting a professional definition of nursing that includes the nurse as a participant in research as an expectation and not an "extra" will be necessary to lay a foundation for change in this area. This role is supported by Magnet ${ }^{\bullet}$ requirements, and demonstrated in some clinical ladder programs, yet needs additional support from nursing leaders, including educators. Nursing curricula could better emphasize the skills needed for engaging in research.

Many changes in the area of ethics review or approval will be necessary to decrease barriers to multicenter studies in any discipline [16]. Some changes would specifically facilitate nursing research. In one potential site for the SIM City study, IRB paperwork was completed and then the master's prepared nurse was informed she was not eligible to be a PI. Despite having a supportive physician colleague who was willing to serve as PI on this research study, the nurse used the minimal nonclinical time afforded to focus efforts on the creation of system-wide change to support the broadened application of a PI role and to develop an appropriate process of support for nurses in research. It is recommended that nurses work within systems when possible to change SOPs so that nursing research is easier to initiate. There was a frequent perception that IRBs were not supportive to or knowledgeable about nursing research. A smooth process to IRB approval would facilitate and support both novice and experienced researchers. Nurses who are interested in research should work to develop relationships with IRB members, volunteer to sit on IRB committees, and open communication with IRBs to provide education about nursing research to non-nurses.

For the benefit of future nurses interested in research, we concur with the suggestion made by Loera [28] and Vedelo and Lomborg [8] that more nurses publish their research experiences so that others may learn from them and are encouraged by them. Additionally, nurses interested in research should seek mentors and enter into the process with a spirit of persistence. Recommendations for future studies include comparing institutions with formal support for nursing research to those without such support. This may assist in identifying effective interventions to facilitate nurses across the continuum of research participation roles [29].

\section{References}

1. Olson DM, McNett MM, Lewis LS, Riemen KE, Bautista C (2013) Effects of nursing interventions on intracranial pressure. Am J Crit Care. 22: 431-438

2. Olson DM, Lewis LS, Bader MK, Bautista C, Malloy R, et al(2013) Significant practice pattern variations associated with intracranial pressure monitoring. J Neurosci Nurs.45:186-193

3. Nightingale F, Skretkowicz V (1992) Florence Nightingale's Notes on Nursing. Reviewed with additions ed. London: Scutari Press

4. American Nurses Credentialing Center(2012) Magnet Recognition Program Model

5. About the NLN: Priorities for research in nursing education (2012)

6. Atkinson M, Turkel M, Cashy J (2008) Overcoming barriers to research in a Magnet community hospital. J Nurs Care Qual. 23:362-368
7. Woodward V, Webb C, Prowse M. Focus (2007) The perceptions and experiences of nurses undertaking research in the clinical setting. Journal of Research in Nursing.12: 227-244.

8. Vedelø TW, Lomborg K (2011) Reported challenges in nurse-led randomised controlled trials: an integrative review of the literature. Scand J Caring Sci 25: 194-200.

9. Darbyshire P (2008) Children's nurses' research involvement: making practice-focused research happen. J Clin Nurs 17: 3238-3244.

10. Higgins I1, Parker V, Keatinge D, Giles M, Winskill R, et al. (2010) Doing clinical research: the challenges and benefits. Contemp Nurse 35: 171-181.

11. Snelgrove S, James M (2011) Graduate nurses' and midwives' perceptions of research. Journal of Research in Nursing. 16:7-20.

12. Darbyshire P (2008) Children's nurses' research involvement: making practice-focused research happen. J Clin Nurs 17: 3238-3244.

13. Lindquist R, Treat-Jacobson D, Watanuki S (2000) A case for multisite studies in critical care. Heart Lung 29: 269-277.

14. Flynn L (2009) The benefits and challenges of multisite studies: lessons learned. AACN Adv Crit Care 20: 388-391.

15. McWilliams R, Hoover-Fong J, Hamosh A, Beck S, Beaty T, et al. (2003) Problematic variation in local institutional review of a multicenter genetic epidemiology study. JAMA 290: 360-366.

16. Gold JL, Dewa CS (2005) Institutional review boards and multisite studies in health services research: is there a better way? Health Serv Res 40: 291-307.

17. U.S.F.D.A. Using a Centralized IRB Review Process in Multicenter Clinical Trials. 2006

18. Loera GM (2006) Experiencing the barriers of nursing research. Gastroenterol Nurs 29: 251-253.

19. Schmelzer M1, Daniels G, Dudley-Brown S, Foote K, Hayes A, et al. (2011) Multisite research: a spirit of cooperation. Gastroenterol Nurs 34: 327-330.

20. CDC (2008) CDC Study Finds that Adoption of the Brain Trauma Foundation Guidelines Could Result in a Substantial Reduction in Traumatic Brain-Injury-Related Deaths.

21. Bratton SL, Chestnut RM, Ghajar J, McConnell Hammond FF, Harris $\mathrm{OA}$, et al. Guidelines for the management of severe traumatic brain injury. VI. Indications for intracranial pressure monitoring. Journal of neurotrauma.24 Suppl 1:S37-44.

22. Hesdorffer DC, Ghajar J, Iacono L (2002) Predictors of compliance with the evidence-based guidelines for traumatic brain injury care: a survey of United States trauma centers. J Trauma 52: 1202-1209.

23. Olson DM, Bader MK, Dennis C, Mahanes D, Riemen K (2010) Multicenter pilot study: safety of automated chest percussion in patients at risk for intracranial hypertension. J Neurosci Nurs 42: 119-127.

24. Streiner D, Sidani $S(2011)$ When research goes off the rails: why it happens and what you can do about it: Guilford Press

25. Balakas K, Bryant T, Jamerson P (2011) Collaborative research partnerships in support of nursing excellence. Nurs Clin North Am 46: 123-128.

26. Woodward V, Webb C, Prowse M (2007) The perceptions and experiences of nurses undertaking research in the clinical setting. Journal of Research in Nursing. 12: 227-44

27. Atkinson M, Turkel M, Cashy J (2008) Overcoming barriers to research in a Magnet community hospital. J Nurs Care Qual 23: 362-368.

28. Loera GM (2006) Experiencing the barriers of nursing research. Gastroenterol Nurs 29: 251-253.

29. Boase S1, Kim Y, Craven A, Cohn S (2012) Involving practice nurses in primary care research: the experience of multiple and competing demands. J Adv Nurs 68: 590-599. 